\title{
On the quality of acoustical measures when evaluating fruits quality
}

T. Tiplica ${ }^{1, \star}$, S. Verron ${ }^{1}$, C. Grémy-Gros ${ }^{1}$, P. Vandewalle ${ }^{2}$, and E. Mehinagic ${ }^{2}$

1 UNAM Université, Laboratoire Angevin de Recherche en Ingénierie de Systèmes (LARIS), University of Angers, France

${ }^{2}$ Groupe de Recherche en Agro-industrie sur les Produits et les Procédés (GRAPPE), Ecole Supérieure d'Agriculture Angers, France

Received: 16 February 2015 / Accepted: 19 May 2015

\begin{abstract}
In this article we show that, without any technological modification, an acoustic firmness sensor used for estimating fruits firmness, can also be used in order to discriminate between different apple varieties. A repetability and reproducibility $(R \& R)$ analysis shows that the device is capable to measure not just the mass and the resonance frequency of fruits but also some other key spectral characteristics as well. Thus, 18 key features were identified and used for the classification of apples belonging to 10 different varieties. The prospects of this study are interesting in the context of sorting automatically fruits.
\end{abstract}

Keywords: Capability study, acoustic measure, spectral analysis, classification, non-destructive test

\section{Nomenclature}

\begin{tabular}{ll}
\hline Symbol & Name \\
\hline$A_{\max }$ & Maximum amplitude \\
$A_{\min }$ & Minimum amplitude \\
$C$ & Class \\
$F_{\max }$ & Frequency corresponding to $A_{\max }$ \\
$F_{\min }$ & Frequency corresponding to $A_{\min }$ \\
$k$ & Number of descriptors \\
$m$ & Mass (kg) \\
$n$ & number of observations \\
$p$ & Dimension of the observation $x$ \\
$P$ & Probability \\
$x$ & Observation \\
\hline
\end{tabular}

\section{Introduction}

Elaborating simple, reliable and rapid non destructive systems to evaluate and guarantee the quality of fruits is nowadays an important research topics. In the last decades, different acoustic techniques have been developed in order to measure the quality of fruits: firmness, external and internal defaults, diseases, changes during the shelf life, etc. Thus, acoustic techniques have been applied for measuring the firmness of several fruits such as apples [1], pears [2], melons [3], kiwifruits [4], tomatoes [5], pineapples [6] and mandarins [7]. Acoustic measurements have also allowed to identify external defects on egg [8] or pistachio [9] and internal defaults in pears [10], watermelons [11], cheese [12] and potatoes [13]. The use of acoustic impulse response is increasing as a valuable tool to evaluate changes during harvest and postharvest [14].

^ Correspondence: teodor.tiplica@univ-angers.fr
Acoustic waves can be transmitted, reflected, refracted or diffracted as they interact with the material. The velocity of the wave propagation, its attenuation and reflection are important parameters used to evaluate tissue properties. Elastic properties of biological tissues gave a vibrational behavior, which is used as an indicator of their internal characteristics [15]. Several non-destructive techniques were developed to observe this behavior: (1) the product may be hit with a small hammer [16]; (2) dropping onto an impact plate [17] and, (3) submitted to an ultrasonic pulser [18] or laser Doppler vibrometer [19].

Different acoustic parameters have been related to the fruits quality (i.e. first resonance frequency, maximum amplitude or band magnitude).

Most of previous studies have concentrated their efforts on few particular points of the acoustic signal (dominant frequency, maximum amplitude or band magnitude). We belive that acoustic signals may contain more information about the physical or chemical properties of fruits than the single firmness. In this study, we use the global acoustic signal in order to identify some relevant characteristics for the discrimination of 10 apples varieties. Neverthless, before using them for classification purposes, a repetability and reproducibility (R\&R) study was conducted in order to assess the capability of the measurement system. This is an important aspect that is unfortunately very often ignored when conducting experimental strategies. Yet it is important to know the amount of variation that is intrinsic to fruit and compare it with that due to the measurement system. The quality of the data obtained from measurement systems should be a constant concern of researchers, engineers and operators. If data have poor quality, even with the best statistical models 

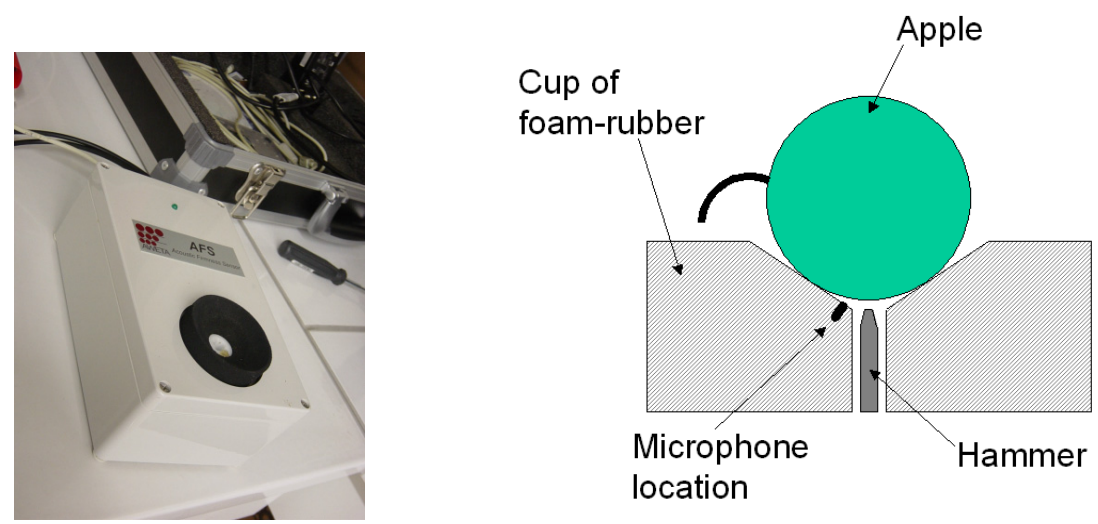

Fig. 1. The experimental device (AFS, Aweta).

the expected results may not be achieved. It is somehow as if one would like to build castles on sand... Without a solid foundation any ediffice is brought to collapse. This is the main motivation that led us to check the measurement results prior to exploit them in order to build a statistical model used for the discrimination of varieties of apples.

\section{Materials and methods}

\subsection{Biological material}

Ten apple varieties (Antares, Ariane, Braeburn, Cameo, Elstar, Jubile, Pink Lady, Reineta Armonique, Red Delicious, Temptation) harvested at optimal commercial maturity on the experimental station (la Station Fruitière de La Morinière - France) was studied. The choice of these 10 varieties has been motivated by the following considerations: fruit size and form were variable, and these fruits presented different textural attributes.

\subsection{Description of our experimental device}

In this study, we used an acoustic firmness sensor (AFS) commercialized by AWETA B.V. (Nootdorp, The Netherlands). This device uses an electromagnet-driven hammer to hit the fruit, a small microphone to capture the resulting acoustic signal and a weight cell (see Fig. 1). The hammer is very light and its extremity is spherical so the fruit is not damaged after the impact. The acoustic signal resulting after the impact of the hammer on the fruit is registered and can be processed by a Fast Fourier Transform (FFT) in order to obtain the power spectrum of the signal. The signal processing is done by a MATLAB software routine. Then, the resonance frequency is identified and the stiffness coefficient is calculated.

\subsection{Evaluation of the capability of the AWETA FMS device}

Each measurement performed by a measuring system has two sources of variation: the inherent variation in the measured characteristic and the inherent variation of the measurement system. The R\&R study is required to evaluate the variation due to the gage and the variation due to operators. Repeatability, called also "Equipment Variation" (EV), is the variability observed when an operator measures several times the same part with the same gage. The reproducibility, called also "Appraiser Variation" (AV), is the additional variation when several operators measure the same part with the same gage. Adding these two sources of variation gives the $R \& R$ variability of the gage:

$$
\sigma_{\mathrm{R} \& \mathrm{R}}^{2}=\sigma_{\text {Repeatability }}^{2}+\sigma_{\text {Reproducibility }}^{2}
$$

Therefore, the total variability of a measured characteristic can be expressed as:

$$
\sigma_{\text {Total }}^{2}=\sigma_{\text {Characteristic }}^{2}+\sigma_{\mathrm{R} \& \mathrm{R}}^{2}
$$

It is common to express the different sources of variation as an interval containing $99 \%$ of the theoretical distribution of the measurement results (that is to say $5.15 \sigma$ ). Thus, $E V=5.15 \sigma_{\text {Repeatability }}, A V=5.15 \sigma_{\text {reproducibility }}$ and $R \& R=5.15 \sigma_{\mathrm{R} \& \mathrm{R}}$.

When expressing the variation of $R \& R$ as a percentage of the tolerance interval (TI) (see Eq. (3)), or as a percentage of the total variability of the measured characteristic (see Eq. (4)) one obtains a capability index which can be used in order to assess the the ability of the measuring system to do the required measurements.

$$
\begin{aligned}
& C=\frac{\sigma_{\mathrm{R} \& \mathrm{R}}}{\sigma_{\text {Total }}} \times 100 \% \\
& C=\frac{5.15 \sigma_{\mathrm{R} \& \mathrm{R}}}{T I} \times 100 \%
\end{aligned}
$$

Usually the criteria used to assess the ability of a measuring system to carry out the measures are:

$C \leqslant 20 \% \quad$ The measuring system is capable.

$20 \%<C \leqslant 30 \%$ The measuring instrument may be used but with caution.

$30 \%<C \quad$ The measuring system is not capable. 


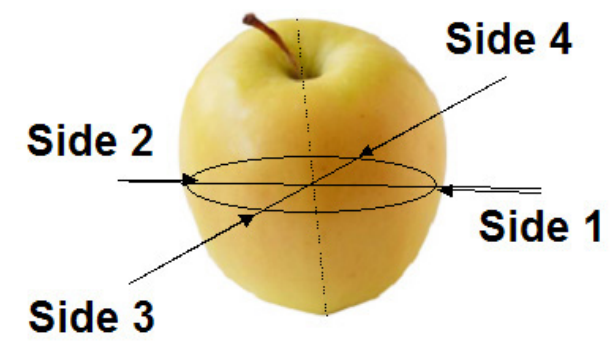

a)

Fig. 2. (a) The measured sides of apples and (b) the median spectra used for apples classification.

b)

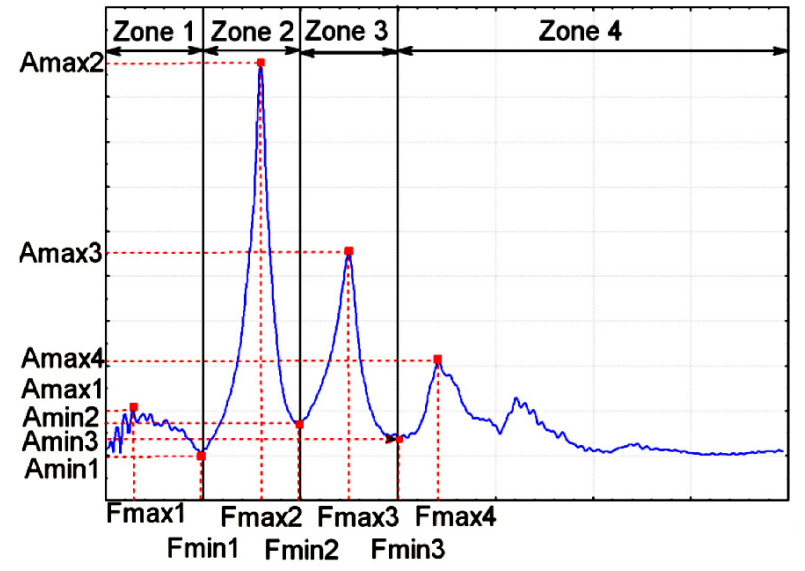

Fig. 3. Retained characteristic points of the spectral curve of an apple.

When conducting a R\&R study other potential sources of variation are considered negligible. This does not mean that the calibration, the stability or the linearity are less important, but their impact on the results is considered to be negligible.

For more information concerning the fundamentals of $\mathrm{R} \& \mathrm{R}$ studies the reader is invited to consult the following references [20,21].

We conducted a $R \& R$ study in order to evaluate the capability of the gage to measure the mass of the fruits and four main frequencies $\left(F_{\max _{1}}, F_{\max _{2}}, F_{\max _{3}}, F_{\max _{4}}\right)$ corresponding to the four highest amplitudes in the spec$\operatorname{tra}\left(A_{\max _{1}}, A_{\max 2}, A_{\max _{3}}, A_{\max _{4}}\right)$ as illustrated in Figure 3 . Ten apples of Golden variety were measured by three operators with the AWETA AFS device. Ten measurements were done on each of four sides of the fruit: on the sun face, the face opposite to that exposed to the sun (or shade side) and the two intermediary sides (Fig. 2a). In this way 40 measurements per fruit were collected. For the R\&R study we used two median spectra: the first median spectrum was computed on the first five measures on each side of the fruits and the second median spectrum was computed using the last five measures. Thus, each median spectrum was computed by using $5 \times 4=20$ spectral characteristics. The spectral range corresponding to

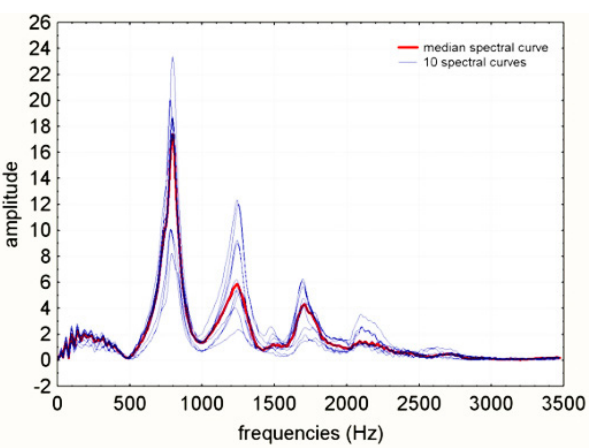

low frequencies (between 0 and $3500 \mathrm{~Hz}$ ) was explored because this range is most informative about the different characteristics of a given variety.

\subsection{Discriminant analysis}

Two different aspects related to this statistical technique are very important: the descriptive aspect (how to separate maximally the $k$ identified classes in the data structure) and the predictive aspect (how to allocate a new observation to one of the $k$ identified classes in the data structure). In the remaining part of this article, we will focus mainly on the predictive potential of the discriminant analysis and we will consider discriminant analysis as a supervised classification method [22].

The Bayes rule is used in the frame of the discriminant analysis method in order to allocate for a new observation $x$ the class $C_{i}$ with the maximal a posteriori probability:

$$
P\left(C_{i} \mid x\right)=\frac{P\left(C_{i}\right) P\left(x \mid C_{i}\right)}{P(x)}
$$

where: $P\left(C_{i}\right)$ is the a priori probabilities of the class $C_{i}$, $P\left(x \mid C_{i}\right)$ is the probability of the observation $x$ given the class $C_{i}$ and $P(x)$ is the probability of the observation $x$.

In this study we considered the same a priori probabilities for each apple vaieties because the sample size is the same in each case (10 apples/variety).

According to the way that separation boundaries between classes are constructed discriminant analysis can be linear (the separation of classes is done by some hyperplanes) or quadratic (non-linear separation boundaries are constructed to separate the classes).

For more information concerning the discriminant analysis technique, the reader is invited to consult the following references $[23,24]$.

In this study, the canonical discriminant analysis was done with MATLAB ${ }^{\circledR}$ software.

We used the information present in the frequency spectrum of the acoustic signal and retained for apples varieties discrimination the median spectra that is most representative for the repeated tests on the same side of 
Table 1. The capability results for the balance.

\begin{tabular}{lcccc}
\hline & $\begin{array}{c}\text { Standard } \\
\text { deviation }\end{array}$ & Variance & \% of R\&R & \% of Total \\
\hline Repetability & 0.78 & 0.61 & 54.93 & 0.25 \\
Reproducibility & 0.71 & 0.50 & 45.07 & 0.20 \\
Apple-to-Apple variation & 15.77 & 248.55 & & 99.55 \\
R\&R variability & 1.06 & 1.12 & 100.00 & 0.45 \\
Total & 15.80 & 249.66 & & 100.00 \\
\hline
\end{tabular}

the same fruit (Fig. 2b). We identified fourteen key spectral characteristics in order to better describe the varietal differencies between apples (Fig. 3):

- $A_{\max _{1}}, A_{\max _{2}}, A_{\max _{3}}, A_{\max _{4}}$ - amplitudes of the first, second, third and fourth pikes of the apples' spectra.

- $F_{\max _{1}}, F_{\max _{2}}, F_{\max _{3}}, F_{\max _{4}}$ - the frequencies corresponding to $A_{\max _{1}}, A_{\max _{2}}, A_{\max _{3}}$ and $A_{\max _{4}}$.

- $A_{\min _{1}}$ - the amplitude of the minimum between $A_{\max _{1}}$ and $A_{\max _{2}}$.

- $A_{\min _{2}}$ - the amplitude of the minimum between $A_{\max _{2}}$ and $A_{\max _{3}}$.

- $A_{\min _{3}}$ - the amplitude of the minimum between $A_{\max _{3}}$ and $A_{\max _{4}}$.

- $F_{\min _{1}}, F_{\min _{2}}, F_{\min _{3}}$ - the frequencies corresponding to $A_{\min _{1}}, A_{\min _{2}}$ and $A_{\min _{3}}$.

Moreover four additional parameters called Energy 1, Energy 2, Energy 3 and Energy 4, corresponding to the areas of four zones demarcated as in Figure 3, were calculated. These 18 parameters were used as descriptors for the quadratic discriminant analysis to classify the fruits according to their varieties.

When analysing classifiers performances, two important caracteristics are: the generalisation ability of the classifier and it's robustness.

The generalisation represents the ability of the classifier to correctly classify observations that have not participated in the construction of the discriminant functions. The generalization ability of the classifier was evaluated through the cross validation test. The "leave one out" technique is very effective and often used in crossvalidation of different classifiers. The principle of this technique is to leave out one observation for the test and to use the remaining observations for the construction of the discriminating functions. This operation is repeated for each observation present in the data set and then, the mean of the different classification errors is calculated. In this way one obtains a very good estimation of the real classification error rate of the classifier.

The robustness of a classifier is related to the number of descriptors included in the model. It is well-known that keeping non-informative descriptors in the model increases the classification error rate of new observations. The final goal when using a classifier is to obtain the lowest classification error of observations which did not participate to the construction of discriminant functions with the smallest number of descriptors [25].

\section{Results and discussion}

\subsection{Capability of the AWETA AFS mesuring system}

As illustrated in Figure 2b a fairly large variability exists in spectra for repeated measures on the same side of the same fruit. This variability occurs predominantly at the level of spectral amplitudes and it is less pronounced at the frequencies level. The question that arise here is to know how much of this variability is due to the measuring system and how much is inherent to non-homogeneities of the internal behaviour of apples. The R\&R study offer an answer to this question.

\subsubsection{The capability of the mass measuring system}

Table 1 show the results we obtained when evaluating the capability of the balance to measure the mass of the fruits. It can be seen that the repetability and reproducibility variation represents only $0.45 \%$ of the total variation enregistered. The repeatability and the reproducibility variations are very similar $54.93 \%$ of the $\mathrm{R} \& \mathrm{R}$ variation comes from the gage and $45.07 \%$ of the $R \& R$ variation comes from the operators). The capability index of the mass measuring system is $0.45 \%<20 \%$ which means that the mass measuring system is able to do the required measurements. This conclusion is reinforced when looking at the $\bar{X}$ control chart for means where it can be seen that the three paterns coresponding to the three operators are very similar (see Fig. 4a). This indicates a good agreement between the operators measurements which means that the operators haven't any dificulties to do the measurments (see also the range control chart given in Fig. 4b). A large majority of points on the $\bar{X}$ control chart falls outside of the interval defined by the two control limits which means that the balance is able to distinguish the differencies between the mass of various measured fruits. Neverthless, it can be observed on the R\&R box-plot (see Fig. 4c) that Operator's 1 average is higher that those of two other operators. Thus, an improvement of the mass measurement system capability can be obtained if the calibration of the balance is done prior to operator changement.

\subsubsection{The capability of the acoustic sensor}

Table 2 show the results we obtained when evaluating the capability of the acoustic sensor to measure the four main 


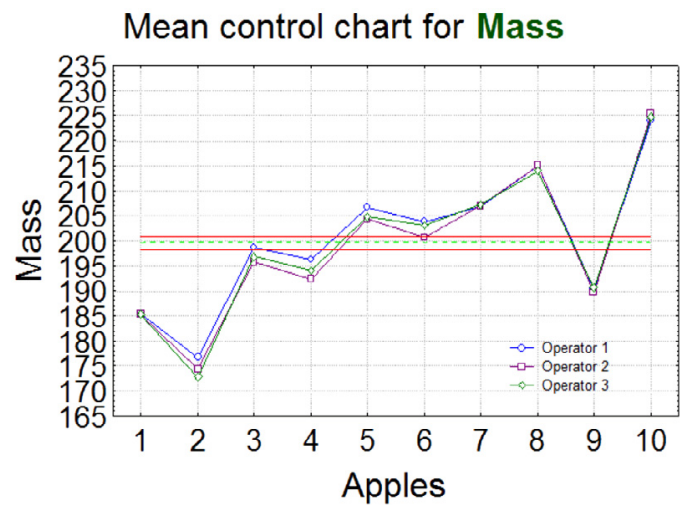

(a)

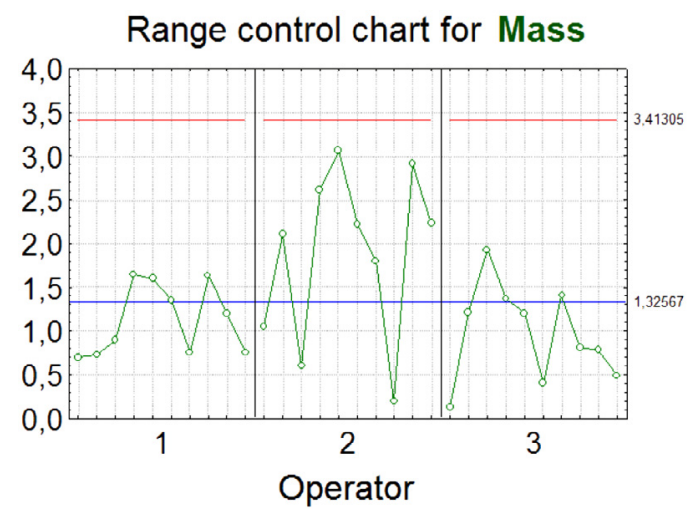

(b)

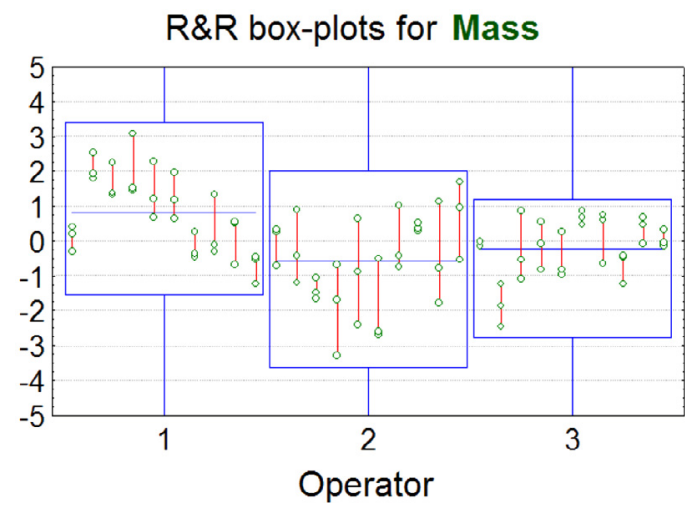

(c)

Fig. 4. (a) $\bar{X}$, (b) range control charts and (c) R\&R box-plots for the mass measurements.

frequencies as indicated in Section 2.3. All the capability indexes are less than $20 \%$ which means that the acoustic firmness sensor is capable to measure these key frequencies in the spectra of the acoustic signal. It can be noticed that the best capability index coresponds to the resonance frequency $\left(F_{\max _{2}}\right)$ of the fruit. Figure 5 shows the main results coresponding to the resonance frequency (similar results have been obtained for $F_{\max _{1}}, F_{\max _{3}}$ and $\left.F_{\max _{4}}\right)$. Some isolated repeatability problems can be noticed for each operator, but we didn't find a sufficient explanation for them.

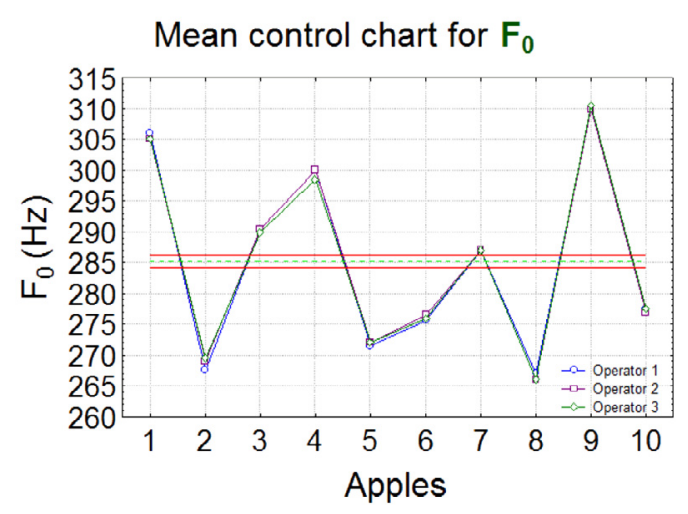

(a)

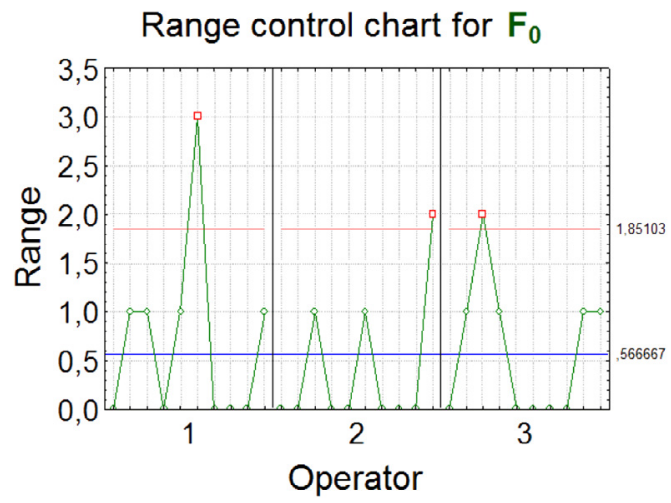

(b)

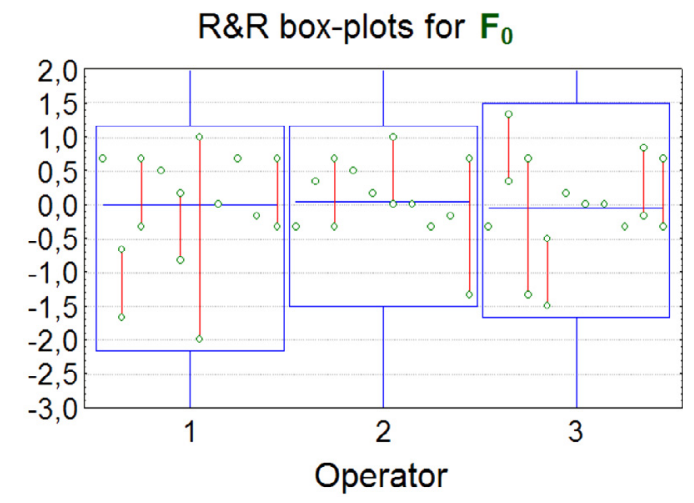

(c)

Fig. 5. (a) $\bar{X}$, (b) range control charts and (c) R\&R box-plots for the resonance frequency measurement.

It can be noticed that excepting the amplitude $A_{\text {max }_{4}}$ for which the capability index is $27.55 \%$, the AWETA AFS device is able to measure amplitudes properly (all the capability indexes are less than $20 \%$ - see Tab. 3). Neverthless, the variability due to the gage is higher than the variability due to the operators. One possible explanation for this phenomenon could be the impact force of the hammer which is not constant from one measurement to other. We think that the measurement device can be improved by the regulation of the impact force of the hammer. 
Table 2. The capability results for frequencies in the acoustic spectra.

\begin{tabular}{|c|c|c|c|c|c|}
\hline & & Standard deviation & Variance & $\%$ of $\mathrm{R} \& \mathrm{R}$ & $\%$ of Total \\
\hline \multirow{5}{*}{$F_{\max _{1}}$} & Repetability & 3.958 & 15.67 & 92.823 & 2.521 \\
\hline & Reproducibility & 1.101 & 1.212 & 7.177 & 0.195 \\
\hline & Apple-to-Apple variation & 24.588 & 604.573 & & 97.284 \\
\hline & R\&R variability & 4.109 & 16.881 & 100 & 2.716 \\
\hline & Total & 24.929 & 621.454 & & 100 \\
\hline \multirow{5}{*}{$F_{\max _{2}}$} & Repetability & 0.502 & 0.252 & 100 & 0.132 \\
\hline & Reproducibility & 0 & 0 & 0 & 0 \\
\hline & Apple-to-Apple variation & 13.788 & 190.114 & \multirow{3}{*}{100} & 99.868 \\
\hline & $\mathrm{R} \& \mathrm{R}$ variability & 0.502 & 0.252 & & 0.132 \\
\hline & Total & 13.797 & 190.366 & & 100 \\
\hline \multirow{5}{*}{$F_{\max _{3}}$} & Repetability & 3.072 & 9.439 & 100 & 0.847 \\
\hline & Reproducibility & 0 & 0 & 0 & 0 \\
\hline & Apple-to-Apple variation & 33.238 & 1104.794 & \multirow{3}{*}{100} & 99.153 \\
\hline & $\mathrm{R} \& \mathrm{R}$ variability & 3.072 & 9.439 & & 0.847 \\
\hline & Total & 33.38 & 1114.233 & & 100 \\
\hline \multirow{5}{*}{$F_{\max _{4}}$} & Repetability & 2.718 & 7.386 & 100 & 1.357 \\
\hline & Reproducibility & 0 & 0 & 0 & 0 \\
\hline & Apple-to-Apple variation & 23.173 & 536.967 & \multirow{3}{*}{100} & 98.643 \\
\hline & R\&R variability & 2.718 & 7.386 & & 1.357 \\
\hline & Total & 23.331 & 544.353 & & 100 \\
\hline
\end{tabular}

Table 3. The capability results for amplitudes in the acoustic spectra.

\begin{tabular}{|c|c|c|c|c|c|}
\hline & & Standard deviation & Variance & $\%$ of $\mathrm{R} \& \mathrm{R}$ & $\%$ of Total \\
\hline \multirow{5}{*}{$A_{\max _{1}}$} & Repetability & 0.0003 & 0.0000 & 99.72 & 12.68 \\
\hline & Reproducibility & 0.0000 & 0.0000 & 0.28 & 0.04 \\
\hline & Apple-to-Apple variation & 0.0007 & 0.0000 & & 87.29 \\
\hline & $\mathrm{R} \& \mathrm{R}$ variability & 0.0003 & 0.0000 & 100 & 12.71 \\
\hline & Total & 0.0008 & 0.0000 & & 100 \\
\hline \multirow{5}{*}{$A_{\max _{2}}$} & Repetability & 0.0004 & 0.0000 & 97.18 & 7.47 \\
\hline & Reproducibility & 0.0001 & 0.0000 & 2.82 & 0.22 \\
\hline & Apple-to-Apple variation & 0.0014 & 0.0000 & \multirow{3}{*}{100} & 92.31 \\
\hline & $\mathrm{R} \& \mathrm{R}$ variability & 0.0004 & 0.0000 & & 7.69 \\
\hline & Total & 0.0015 & 0.0000 & & 100 \\
\hline \multirow{5}{*}{$A_{\max _{3}}$} & Repetability & 0.0002 & 0.0000 & 92.46 & 16.77 \\
\hline & Reproducibility & 0.0000 & 0.0000 & 7.54 & 1.37 \\
\hline & Apple-to-Apple variation & 0.0004 & 0.0000 & \multirow{3}{*}{100} & 81.86 \\
\hline & R\&R variability & 0.0002 & 0.0000 & & 18.14 \\
\hline & Total & 0.0004 & 0.0000 & & 100 \\
\hline \multirow{5}{*}{$A_{\max _{4}}$} & Repetability & 0.0002 & 0.0000 & 61.95 & 17.07 \\
\hline & Reproducibility & 0.0001 & 0.0000 & 38.05 & 10.48 \\
\hline & Apple-to-Apple variation & 0.0003 & 0.0000 & \multirow{3}{*}{100} & 72.45 \\
\hline & R\&R variability & 0.0002 & 0.0000 & & 27.55 \\
\hline & Total & 0.0004 & 0.0000 & & 100 \\
\hline
\end{tabular}

\subsection{Discrimination of apples varieties}

The classification error obtained by taking into account all 400 measures (i.e. 10 fruits/variety $\times 10$ varieties $\times$ 4 faces/fruit) is $0.25 \%$. A single observation was erroneously classified - an apple that belongs to the Cameo variety was classified as a Red Delicious apple. This result is very encouraging because it shows that each kind of fruit has its own spectral signature, and that it is possible to distinguish the fruit variety from some key features of it's spectrum.

In order to obtain a more realistic estimation of the efficiency of the quadratic discriminant analysis, we evaluated the generalization ability and the robustness of this classifier. In our case, by using the "leave one out" cross validation technique the classification error was found equal to $14.75 \%$.

In Figure 6 one can see the classification error obtained when varying the number of descriptors from 1 to 18. These results have been obtained by "leave one out" cross-validation technique. At the beginning, the classification error decreases very fast with the increase of the number of descriptors, but starting from 10 descriptors the slope of the decreasing tendency is small. It can be noticed also that the percentage of misclassification is underestimated when evaluated on the complete data. 
Table 4. Statistical significance of the descriptors.

\begin{tabular}{ccccccc}
\hline & Wilk $(\lambda)$ & Partial $(\lambda)$ & $F_{\text {exclusion }}(2.58)$ & $p$ level & Tolerance & 1-Tolerance $\left(R^{2}\right)$ \\
\hline$A_{\max _{1}}$ & 0.006 & 0.792 & 10.827 & 0.000 & 0.119 & 0.880 \\
$F_{\max _{1}}$ & 0.007 & 0.683 & 19.156 & 0.000 & 0.058 & 0.941 \\
$A_{\max _{2}}$ & 0.006 & 0.826 & 8.684 & 0.000 & 0.086 & 0.913 \\
$F_{\max _{2}}$ & 0.005 & 0.933 & 2.958 & 0.002 & 0.865 & 0.134 \\
$A_{\max _{3}}$ & 0.007 & 0.664 & 20.959 & 0.000 & 0.193 & 0.806 \\
$F_{\max _{3}}$ & 0.005 & 0.923 & 3.442 & 0.000 & 0.030 & 0.969 \\
$A_{\max _{4}}$ & 0.006 & 0.840 & 7.851 & 0.000 & 0.155 & 0.844 \\
$F_{\max _{4}}$ & 0.005 & 0.976 & 0.976 & 0.459 & 0.258 & 0.741 \\
$A_{\min _{1}}$ & 0.005 & 0.954 & 1.960 & 0.042 & 0.336 & 0.663 \\
$F_{\min _{1}}$ & 0.006 & 0.772 & 12.234 & 0.000 & 0.181 & 0.818 \\
$A_{\min _{2}}$ & 0.006 & 0.744 & 14.243 & 0.000 & 0.199 & 0.800 \\
$F_{\min _{2}}$ & 0.006 & 0.734 & 14.963 & 0.000 & 0.055 & 0.944 \\
$A_{\min _{3}}$ & 0.005 & 0.898 & 4.702 & 0.000 & 0.155 & 0.844 \\
$F_{\min _{3}}$ & 0.005 & 0.932 & 2.991 & 0.001 & 0.027 & 0.972 \\
Energy 1 & 0.006 & 0.783 & 11.436 & 0.000 & 0.138 & 0.861 \\
Energy 2 & 0.006 & 0.760 & 13.038 & 0.000 & 0.126 & 0.873 \\
Energy 3 & 0.007 & 0.704 & 17.358 & 0.000 & 0.279 & 0.720 \\
Energy 4 & 0.007 & 0.700 & 17.711 & 0.000 & 0.243 & 0.756 \\
\hline
\end{tabular}

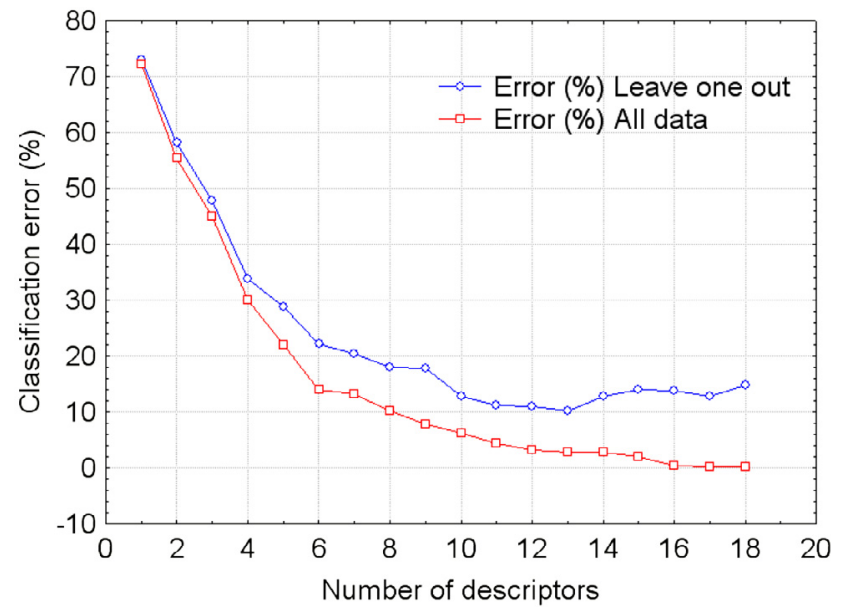

Fig. 6. Influence of the number of descriptors on the classification error rate.

This is more evident as the number of descriptors is important. For the quadratic discriminant analysis, the lowest error rate is obtained for 13 descriptors (10.25\%).

In Table 4, the result of an ANOVA (Analysis of the Variance) test for the 18 descriptors used is given. This test is designed to demonstrate whether a descriptor included in the model is significant from a statistical point of view $[19,23]$. In order to do that, the ratio of two variances ( $F=$ the variance explained by the descriptor/the residual variance which is not explained by the descriptor) is compared with the quartile of the Ficher's law (noted by " $F$ exclusion"). In our case, for a risk $\alpha=5 \%$, " $F$ exclusion" is 2.58. It can be noticed that the amplitude of the third maximum on the spectral curve is the most discriminative descriptor $(F=20.96)$, then follow in order $F_{\max _{1}}$, Energy 4 and Energy 3, etc.

\section{Conclusions and outlook}

This study is a first step in a larger program which aims to assess the quality of apples by non-destructive tests. A consistent database containing information from different sensorial tests and destructive test already exists. It is well-understood that each test provides a wealth of information. The goal is to extract the maximum information on fruit quality without destroying the fruit and without relying on expensive laboratory methods which require much time and dedicated resources. At this stage of the program developpement, we chosed to evaluate the discrimination potential of the acoustic measurements and we worked for this study only on apples.

We showed that without any technological modifications it is possible to use the Acoustic Firmness Sensor developped by AWETA B.V. in order to classify the apples according to theirs varieties. A capability study showed that the measuring system is capable to measure the stiffness of apples. Indeed, the mass of fruits and their resonance frequency are very well measured by the gage. This is not surprising knowing the gage was designed to measure the stiffness of fruits. But, we showed that with this gage it is also possible to measure correctly other key frequencies in the acoustic spectra of fruits and we proved that the use of 18 carefully chosen spectral characteristics allows to discriminate among 10 different varieties of apples. This means that each type of apple has its own spectral signature permitting to unambiguously identify the belonging varieties of any fruit. Among the applications considered in this work we can mention, for example, the automatic sorting of apples.

We will consider undertaking further studies on the possible correlations that may exist between destructive tests and the acoustical technique. We will also compare 
the performance of the acoustical method with those of other non-destructive testing such as optical and laser tests.

Acknowledgements. Many thanks to our collegues from the Experimental Station La Morinière, who provided us with fruits, and to Dr Anthony SOURICE and his students (ESEO Angers - France), who helped us to develop the acquisition system for our experimental device.

\section{References}

1. J.A. Abbott, D.R. Massie, B.L. Upchurch, W.R. Hruschka, Nondestructive sonic firmness measurement of apples, Trans. Am. Soc. Agric. Eng. 38, 1461-1466 (1995)

2. A.H. Gómez, J. Wang, A.G. Pereira, Impulse response of pear fruit and its relation to Magness-Taylor firmness during storage, Postharvest Biol. Technol. 35, 209-215 (2005)

3. J. Sugiyama, K. Otobe, S. Hayashi, S. Usui, Firmness measurement of muskmelons by acoustic impulse transmission, Trans. Am. Soc. Agric. Eng. 37, 1235-1241 (1994)

4. J.A. Abbott, D.R. Massie, Nondestructive sonic measurement of kiwifruit firmness. J. Am. Soc. Horticult. Sci. 123, 317-322 (1998)

5. B. De Ketelaere, J. De Baerdemaeker, Tomato firmness estimation using vibration measurements, Math. Comput. Simul. 56, 385-394 (2001)

6. H. Chen, J. De Baerdemaeker, Modal analysis of the dynamic behavior of pineapples and its relation to fruit firmness. Trans. Am. Soc. Agric. Eng. 36, 1439-1444 (1993)

7. A.H. Gómez, J. Wang, A.G. Pereira, Firmness of mandarin at different picking dates, Food Sci. Technol. Int. 12, 273-279 (2006)

8. H.-K. Cho, W.-K. Choi, J.-H. Paek, Detection of surface cracks in shell eggs by acoustic impulse method, Trans. Am. Soc. Agric. Eng. 43, 1921-1926 (2000)

9. T.C. Pearson, Detection of pistachio nuts with closed shells using impact acoustics, Appl. Eng. Agric. 17, 249-253 (2001)

10. E. Schrevens, R. De Busscher, L. Verstreken, J. De Baerdemaeker, Detection of hollow pears by tree based modelling on non-destructive acoustic impulse response spectra, in Proc. of the International PostHarvest Science Conference, Taupo, New Zealand, 1996
11. B. Diezma-Iglesias, M. Ruiz-Altisent, P. Barreiro, Detection of internal quality in seedless watermelon by acoustic impulse response, Biosyst. Eng. 88, 221-230 (2004)

12. T. Conde, J.A. Cárcel, J.V. García-Pérez, J. Benedito, Non-destructive analysis of Manchego cheese texture using impact force-deformation and acoustic impulse-response techniques, J. Food Eng. 82, 238-245 (2007)

13. I.E. Elbatawi, An acoustic impact method to detect hollow heart of potato tubers, Biosyst. Eng. 100, 206-213 (2008)

14. W.B. Herppich, S. Landahl, B. Herold, J. De Baerdemaeker, Interactive effects of water status and produce texture - An evaluation of non-destructive methods, in Proc. of the International Conference: Postharvest Unlimited (Leuven, Belgium, 2003)

15. H.L. Clark, W. Mikelson, Fruit ripeness tester. U.S. Patent No. 2277037 (1942)

16. F. Duprat, M. Grotte, E. Piétri, D. Loonis, C.J. Studman, The acoustic impulse response method for measuring the overall firmness of fruit, J. Agric. Eng. Res. 66, 251-259 (1997)

17. T.C. Pearson, A.E. Cetin, A.H. Tewfik, R.P. Haff, Feasibility of impact-acoustic emissions for detection of damaged wheat kernels, Digital Signal Proc. 17, 617-633 (2007)

18. A. Bechar, A. Mizrach, P. Barreiro, S. Landahl, Determination of mealiness in apples using ultrasonic measurements, Biosyst. Eng. 91, 329-334 (2005)

19. M. Taniwaki, M. Takahashi, N. Sakurai, Determination of optimum ripeness for edibility of postharvest melons using nondestructive vibration, Food Res. Int. 42, 137-141 (2009)

20. L.B. Barrentine, Concepts for R\&R studies (American Society for Quality, 2003)

21. Measurement systems analysis, Reference manual (Chrysler Corporation, Ford Motor Company, General Motors Corporation, 1995)

22. R. Duda, P.E. Hart, D.G. Stork, Pattern classification, 2nd ed. (Wiley-Interscience, New York, 2001)

23. J.F. Hair, B. Black, B. Babin, R.E. Anderson, R.L. Tatham, Multivariate data analysis, 6th edn. (Prentice Hall, Upper Saddle River, NJ, 2006, 928p.)

24. G.J. McLachlan, Discriminant analysis and statistical pattern recognition (Wiley-Interscience, Hoboken, NJ, 2004)

25. S. Verron, T. Tiplica, A. Kobi, Fault detection and identification with a new feature selection based on mutual information, J. Process Control 18, 479-490 (2008) 